\title{
From Cronbach to Brunswik: A Conceptual Framework for Personality and Individual Differences Research
}

\author{
Trisha Nowland \\ Macquarie University \\ ACPID Conference Paper 2016
}

Recent personality literature has proposed that Cronbach's generalisability theory offers a substantive ground for the integration of inter-individual differences and intrapersonal process approaches to personality trait research. Generalisability theory has the advantage of maximising psychometric dependability of behavioural measurements, but does not demand reconciliation to the environment that is relevant to the life circumstances of the participant. A conceptual analysis contrasting generalisability theory with Egon Brunswik's conceptual framework for psychology is presented. Recommendations regarding the connection between theory and methodological practice follow, in light of an evolutionary approach to personality and individual differences. An example that highlights the contrast between Cronbachian and Brunswikian approaches is presented, resulting in a recommendation to revisit the concept of ecological validity and representational research design, to better account for what is reflected in study outcomes for personality constructs.

Recent literature states that while there are substantial benefits to be gained from proposals for unification in psychology, there remains some problem with a failure to adequately address the disparate theoretical-epistemological commitments that underpin for example present-day personality theory (Poropat \& Corr, 2015; Wood, Gardner \& Harms, 2014; Cramer et al., 2012). Poropat and Corr (2015) track historical developments in personality theory via distinction between intraindividual and inter-individual difference accounts of personality traits. Research investigating intraindividual process dynamics is described as having roots in the experimental/laboratory methodology attributed historically to Wilhelm Wundt, while inter-individual differences research is recounted as having origins in the correlational hereditary investigations of Frances Galton, which later are adopted in the development of factor analysis, a technique which underpins for example the Big Five Model (BFM) of personality. Personality research is noted as typically grounded in either Wundtian or Galtonian bases, but not both. Further, any attempt to integrate Galtonian and Wundtian research 
remains problematic, as statistical analyses are conducted in incompatible ways. For example, even though there is a growing body of work that explores potential for explanatory neurobiological bases for personality factors such as openness or extraversion, there is no logical reason that any of the statistically derived inter-individually calculated BFM factors should explain biologically-based intraindividual processes in causal or evolutionary terms (Poropat \& Corr, 2015; DeYoung et al., 2010; Wood, Harms, \& Gardner, 2015).

A search for grounds for unification or coherence between inter- and intra-individual differences approaches led Poropat and Corr (2015) to endorse generalisability theory as a unifying framework. Generalisability theory is an ANOVA-based statistical approach to measurement error that works to evaluate dependability of psychometric outcomes (Shavelson \& Webb, 1991). Poropat and Corr (2015, p. 60) claim that the systematised explication of measurement error lends generalisability theory the infrastructure that supports integration of disparate approaches in personality research. This paper examines this claim, and contrasts its outcomes with the benefits of a conceptual framework first set out by Egon Brunswik (1903-1955). The results of the conceptual analysis traversing both proposals suggest that environmental variables remain under-represented in generalisability theory. An example that integrates insights from both approaches is presented, but first we begin by reviewing generalisability theory.

\section{Generalisability theory}

Generalisability theory as first set out by Cronbach, Rajaratnam, and Gleser (1963) addresses the lack of differentiation of source for measurement error in classical test theory (CTT). CTT distinguishes true score from error for a given psychometric outcome, but makes no claim regarding the source, or systematised versus unsystematised nature of error properties (Shavelson \& Webb, 1991). Tracking systematic error, generalisability theory maximises dependability of generalisations made from a study (Poropat \& Corr, 2015). A psychometric outcome is understood as having facets in its composition, which include, for example, item, rater, form, and occasion characteristics, among others (Shavelson \& Webb, 1991). In research design, facets are established for each factor included in a statistical model of the psychological phenomena, and an overall analysis of variation or error, and then the study generalisability, is conducted in light of these facets.

\section{Proposal for generalisability theory}

Poropat and Corr (2015) suggest that generalisability theory offers a framework for the integration of historical personality theories because it affords a systematic network by which the roles of intra-individual differences, the particulars of the research situation, and the influence of social others such as the researcher themselves in the formulation of personality assessments can be analysed for any given assessment scenario. Galtonian or inter-individual difference models such as the BFM are noted 
as aiming at but falling short of describing or explaining phenotypical or endophenotypical characteristics of behaviours that have genetic origins, as the models cannot account for the particulars of the situation within which behaviour occurs. Because of the inclusion of facets that provide a level of granularity about aspects of the research situation, Poropat and Corr (2015) state generalisability theory improves the accuracy of description of psychological phenomena, resulting in better explanatory power. With generalisability theory, for example, an observed score from a self-report administration of scale items may be decomposed into means and effects in the following way (see Arterberry, Martens, Cadigan \& Rohrer, 2014, p. 99-100), with a breakdown of effects visualised in the bottom section of Figure 1:

$$
\begin{aligned}
& \text { observed score }= \\
& \quad \text { mean } \\
& + \text { item effect } \\
& + \text { occasion effect } \\
& + \text { person effect } \\
& +(\text { item } x \text { occasion effect }) \\
& +(\text { person } x \text { occasion effect }) \\
& +(\text { person } x \text { item effect }) \\
& +(\text { person } x \text { item } x \text { occasion effect }) \\
& + \text { residual. }
\end{aligned}
$$

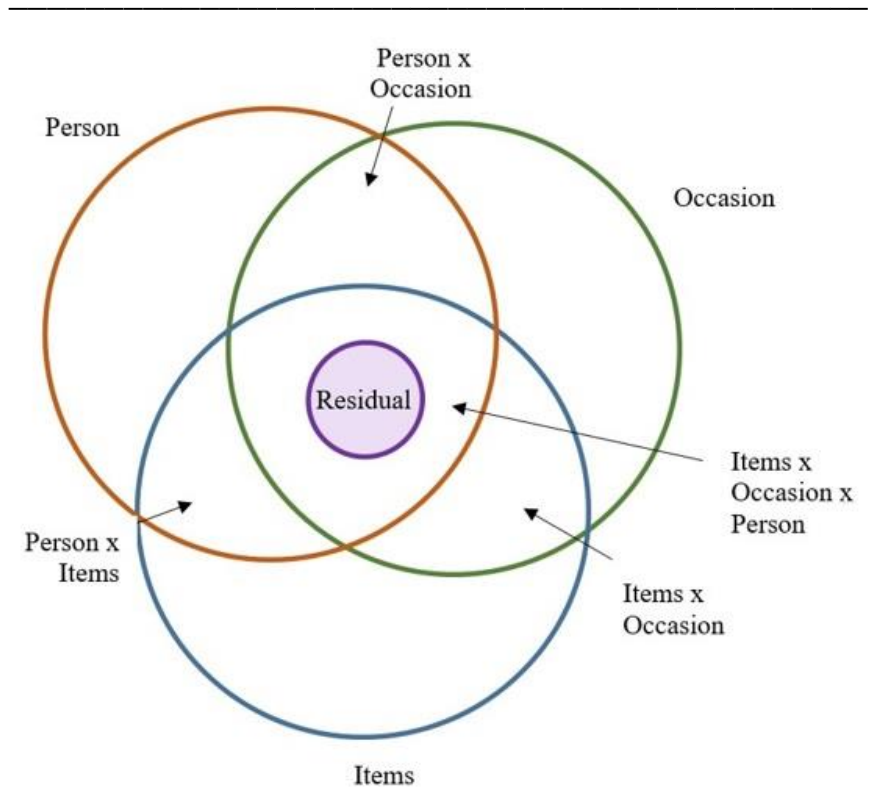

Figure 1 Generalisability theory logic diagram example

The decomposition is possible for any research situation where unique estimated mean 
variances can be identified for facets. A matrix is constructed to estimate facet intersections and examine patterns of systemic variation (Shavelson \& Webb, 1991; see Arterberry et al., 2014, for example). Without such detailed analysis for statistical outcomes, the results of a study remain at best descriptive, rather than either explanatory or predictive, per Poropat and Corr (2015).

\section{Need for conceptual analysis}

Poropat and Corr (2015, p. 68) state that their proposal is one step in the right direction, but also that it falls short of what would best serve unification in personality research, stating that current methods remain overtly simplistic, and that what is needed is an ability to "model the measurement, context, phenotype, endophenotype and genotype of personality simultaneously" (p. 68). Big data presents both an opportunity and a challenge for personality research in this regard. Massive data warehousing lends the opportunity for longitudinal tracking of contextualising data, which serves to maximize the dependability of research outcome as is the aim of generalizability theory, while challenge is presented for personality research in that big data analysis suggests that prediction accuracy is enhanced by utilising online circumstantial behavior as predictors, rather than personality factors (see Kosinski, Stillwell \& Graepel, 2013). This may mean that personality factors may serve optimally in an explanatory, rather than predictive capacity, and that the adopted principles of unification may be defined by what makes a good explanation, over and above what makes a good prediction.

Problems to do with circularity and reification for personality and individual difference factors where they are used in explanatory capacity are set out in Boag (2011). Boag suggests that these problems can be overcome where the biological properties of biological constructs may serve as an explanatory basis for personality factors. Such explanation is robust to the degree that properties associated with the personality factor can be independently asserted without reference to the behaviours that such a personality factor incurs. Notably here we see both reference to biological bases and a logical requirement for the independent referencing of the psychological phenomena of central concern in the explanation - a requirement compatible with Poropat and Corr's (2015) stipulations for unification, above.

Noting the admission of Poropat and Corr (2015), and the logical tests of Boag (2011), it is proposed here that while generalisability theory certainly offers an advance, it falls short of having the cornerstones needed to model genotype and endophenotype accounts of personality, as it misses a reconciliation between the study situation and the environment within which individuals persist, before, during and beyond, the study. It is in this external environment in which expected evolutionary implications for genotypes and endophenotypes will have most import. These environmental cornerstones are supported by adopting the conceptual framework set out by Egon Brunswik (1952), who sought to develop a research methodology informed by probabilistic functionalism, the idea that 
evolution is driven by the ways that organisms respond to their environments, which takes place in a probabilistic manner. We look to conceptual analysis as a guide for what is necessary, when bringing these terms into contact with personality constructs, and research methodology.

\section{Conceptual analysis}

Conceptual analysis remains underutilised, in psychological research, despite longstanding argument for its use (Rozeboom, 1977; Machado \& Silva, 2007; Petocz \& Newbery, 2010). Conceptual analysis takes as object any elements relevant to statements made under the scientific method, which include "concepts, terms, variables, constructs, definitions, assertions, hypotheses, and theories" (Petocz $\&$ Newbery, 2010, p. 126). The process includes clarifying implicit assumptions in arguments and chains of inference, as well as other steps such as resolving semantic ambiguity and examining refutability of hypotheses (Machado \& Silva, 2007; Petocz \& Newbery, 2010). Implicit assumptions about the nature of causation and the role of the environment in the proposal for generalisability theory are discussed in what follows, below.

\section{The gene-behaviour-environment gap}

Poropat and Corr's (2015) proposal for generalisability theory goes some way towards addressing concerns about the explanatory capacity of personality factors, by addressing a level of granularity not typically otherwise tracked in the research situation. The authors maintain however that for those factors calculated on the basis of between-subjects variances, such as the BFM factors, there remain no logical grounds for ascribing a causal or genotype-role for these, to individuals, as there is no one individual reflected in the construction of the model and its factors (see Saucier \& Goldberg, 2001; Lewontin, 2006). Nor can the factor serve in an endophenotypic role (where for example individual attentional processes have import for behavioural expression). What is missed in any regard is reconciliation between the study situation and the environment within which organisms persist outside of the study context. We turn now to examine probabilistic functionalism as an evolutionary theory-informed account, of personality phenomena. Here there is a role for the environment in personality phenomena, as a function of biologically-based organism-environment relationships.

\section{Probabilistic functionalism}

Probabilistic functionalism is a Darwinian approach to psychological phenomena and research methods developed by Egon Brunswik (1939). In common with other more recent proposals (see Petocz \& Mackay, 2013; Marsh \& Boag, 2014), Brunswik (1952) characterises psychological phenomena as situated within organism-environment relations. Brunswik's (1952) conceptual framework is novel in that: i) individual differences in psychological phenomena are understood as 
situated in organism-environment relationships, with the organism and environment individually regarded as a unique and interacting system (Hammond, 1966); ii) it requires that variation in the environment is systematically tracked, with environmental variation considered as at least as important as variation both within and between, individuals.

Brunswik's (1952) probabilistic functionalism places emphasis on an organism's probabilistic sensing of and adaptation to an ever-changing environment, where variation in the environment is at least as influential for psychological phenomena as processes within intra-organism systems, themselves. Beginning in investigations of object-constancy in perception psychology, Brunswik $(1939,1956)$ understood object-constancy as a kind of paradox, where there is not a singular one-to-one relationship between object and perception, rather, the perception-act is a function of complex interaction and mediation processes. These systems or processes have intra-individual as well as organism-to-environment aspects, so that variables may interact or mediate just within the individual, or within the environment, or may interact with and mediate the organismenvironment relation. There are implications then both for events occurring in a given research study, and events shaping how object-perception occurs naturally in the environment, for the organism, which must be addressed in research methodology (Brunswik, 1956). For example, when considering the cue to behaviour for a rat upon perceiving a food- stimulus, experimentally-informed methodology assumes that the food-stimulus is infinitely available. In naturally occurring environments food will occur with probabilistic frequency for a rat, which prompts different cuebehaviour actions, for example, when a food source is exhausted. For Brunswik $(1939,1956)$, this kind of study-environment disparity asked that we play close attention to, and make systematic record of correlations at the level of the environment, and also at the level of the organismenvironment relation, as much as research protocols informed by the Wundtian experimental paradigm were already rigorous in their attention to intra-individual variations, given study manipulations.

In deriving the original form of ecological validity, Brunswik (1952) distinguished between the influence of distal variables, such as those at a geographic or historical distance from the study setting, and proximal ones, which may lend themselves to intervention but upon which the effects of intervention were unpredictable, because of the influence of distal variables. Correlational analyses of environmental impacts upon the organism facilitate the production of a model of the causal texture of the organism's encounter with the environment, such that precise modelling of both systems and their interactions is necessary (Tolman \& Brunswik, 1935). Brunswik (1956) noted, for example, that it was vital to correlate study outcomes over expected environmental changes, for him this was the only way to properly conceive of and account for the evolutionary implications of probabilistic functionalism. For Brunswik $(1939,1952,1956)$ what was vital for the future of psychology was a shift from the organismic focus of systematic design, which had privileged experimental certainty at 
the expense of ecological generalisability, to a representative approach to study design, which accounted for the way in which the environment changed. For Brunswik this meant that at best, psychology could produce probabilistic explanations for its phenomena of interest.

\section{Causation in psychology research}

This account of probabilistic functionalism presents an interesting twist in light of recent accounts of causation relevant to personality factors investigated in factor analytic or latent variable modelling approaches. In "Measuring the Mind: Conceptual Issues in Contemporary Psychometrics", Borsboom (2005) claims the way that psychology researchers use the latent variable model (LVM) in statistical analysis suggests that all such researchers are realist in their commitments, taking between-subjects self-report test scores as caused by an underlying, real, psychological phenomenon or attribute. In this way, for example, extraversion, which is not directly observable is given to cause, self-report test score outcomes that are indirectly measured by the LVM. While the set of assumptions regarding realism and causation for the LVM remain subject to question (see Nowland, 2014; Poropat \& Corr, 2015), and, although Borsboom (2005) himself points to problems with interpreting between-subjects or inter-individual differences as having causal efficacy in the real world, recent developments may have compounded these unresolved dilemmas. The dynamic network model (DNM) has recently been proposed as an advance over the LVM because in the view of its proponents it represents transcendence of problems to do with directionality in causation for latent variables (see Cramer et al., 2012). In the DNM, causation is free to run in any direction, between variables, rather than requiring that the latent variable, such as extraversion, causes observed outcomes (Cramer et al., 2012). Proposing that personality is made up of components of affective, cognitive and behavioural systems which may act causally on each other, Cramer and colleagues suggest that data can freely tell the story about interactions between these systems when utilising the DNM. In this way, for Cramer and colleagues, the DNM represents an advance over the LVM because in their view it steps closer to the 'truth' about causal relationships.

Brunswik's (1956) view of cause and effect in producing psychological phenomena is both more general and more precise than both that of the somewhat permissive DNM, where cause and effect run freely between intra-individual factors, and also compared to the LVM, with its assumption of a one-way casual relation between the factor and scores, calculated on between-subjects data. It is more general as the causal texture of the environment in probabilistic functionalism implies that several different stimuli may be given to cause the same observable outcome. Exhibition of extraversion for example may for some individuals be equally likely in the presence of a single other individual, as it is likely demonstrated at a party, but this is not true for all individuals. In this way, Brunswik's (1952) conceptual framework is more precise, in its account of causation - it claims that what is usually the 'effect' in a causal explanation for psychological phenomena also has a relevant 
causal influence- the environmental event as an 'effect' serves in some ways in a causal role, for psychological phenomena, as well. Causation has clear bi-directionality, and this is the basis of representative design of research, which Brunswik (1956) saw as an advance over experimental design, to the degree that it demands a detailing of the role of the environment in the production of psychological phenomena, or attributes.

Brunswik (1956) states that research methodology should thus include analysis of the effect of subjects encountering the study situation including the self-report scale, in the environment of social relations that make up the fabric of an individual's experience within a cultural context. Conceptually, variation in environments of social relations, or the differences between for example, the three situations of a party, everyday life, and a study context play an important role in what is assessed, in test-score outcomes for personality variables such as extraversion, and this variation must therefore be included in the overall analysis of a personality attribute, within a psychology research study, following Brunswik (1956) (see Figure 2). These variations can be systematically tracked and correlated to produce a modelled version of the causal texture of what it is that individuals encounter, in study and environmental contexts, that produce certain personality phenomena, for observation by researchers. Logically, for Brunswik (1956), without adequate attention to the explanatory generalisability of a study to the environment relevant to the organism or subjects in question, the induction or generalisation from study participants to a broader population as sought in statistical analysis of research outcomes is simply not possible. Generalisation fails because we have not been precise enough in defining the conditions in which say extraversion can be expected to occur. 


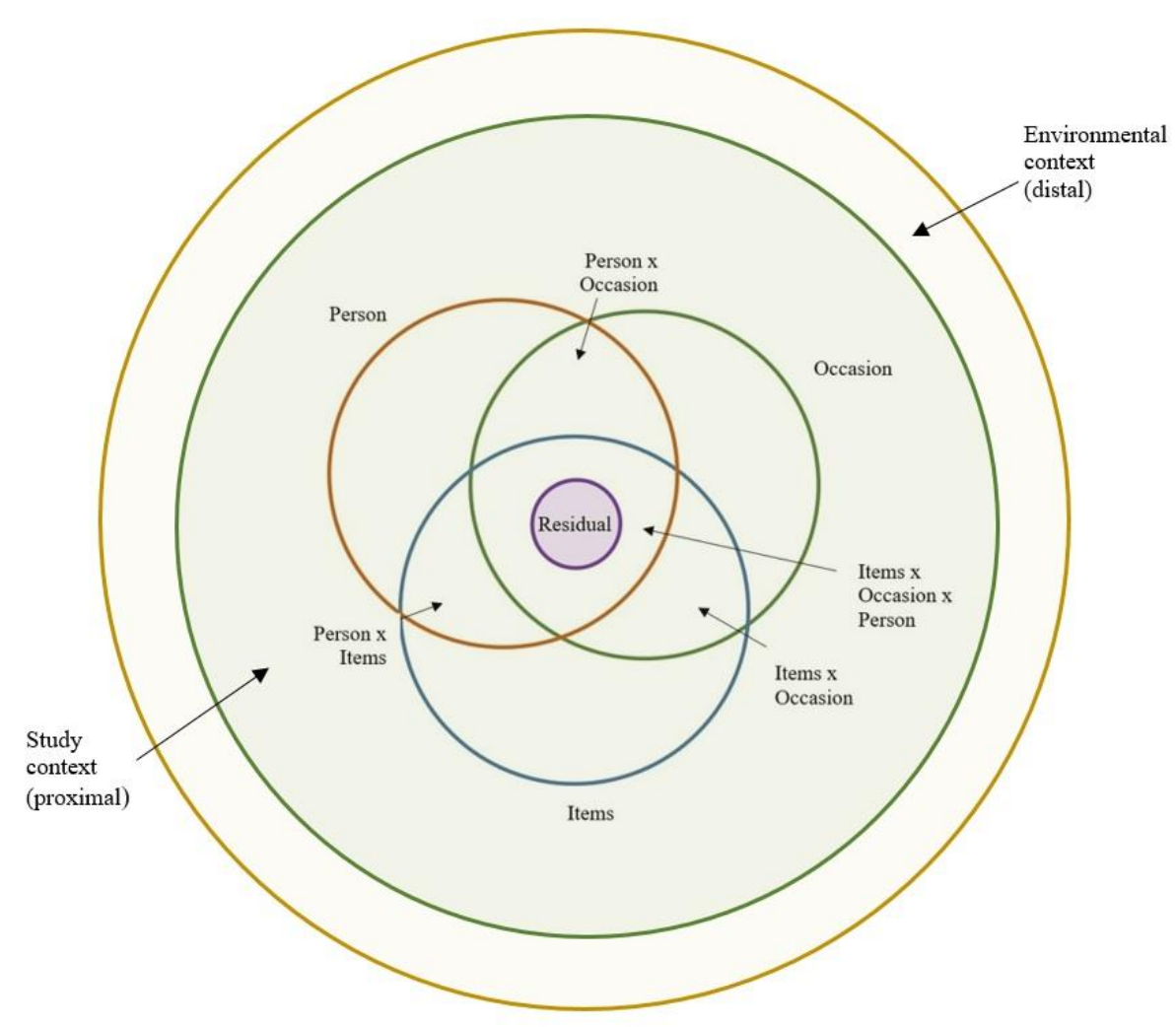

Figure 2. Conceptual framework logic diagram example

\section{Implications for Poropat and Corr's (2015) proposal}

While generalisability theory works with variables internal to a given study structure such as items, persons or occasions of administration, Brunswik's (1956) ecological validity works to extend concerns for generalisability beyond these terms to say that correlational analyses of the organism's environment must also be included in the analysis and reporting of outcomes for psychological research. Not only intra-individual differences, but intra-ecological analysis which maps the study variables to physical-environmental organismic situations is vital to be able to make meaningful statements about the study outcomes in the context of individual differences (Brunswik, 1956; Hammond, 1966). Examples of how this could be done may involve triangulation of observer and self-report test outcomes, with the observer noting individual responses in environmental contexts such as the study setting, and a party setting. It could also involve asking subjects to respond about elements of the environment considered relevant to the attribute or variable in question. In any regard, the effect of any study questionnaire on the individual who fills it out should in some way be represented, in research outcomes. This begins to make room, for example, for cultural differences that may inform perceptions of first-person statements in self-report items (see Saucier \& Goldberg, 2001), or the possibility that for one individual different environments generate different expressions, of personality. 


\section{Recent example}

Arterberry et al. (2015) conducted a generalisability analysis of abbreviated Big Five item scales, on three different time intervals, with 44 total items and then 10 total items ( 2 per factor), across 264 American Midwestern university students, on item, occasion, and person bases. Findings suggested that dependability was enhanced with the larger item set, and that person-by-item variance accounted for the most variance after person variance. Adding insights from Brunswik's (1956) representative design, work prior to the beginning of the participant involvement would include working to estimate stimuli relatedness to environmental circumstances, in this case, perhaps an estimate of an item's meaningfulness in light of the purpose of the administration of the scale, which had formed part of a study of alcohol use, on campus. Contextual elements of psychology research studies have recently been demonstrated to play an important role in reproducibility (see Van Bavel, Mende-Siedlecki, Brady \& Reinero, 2016). Representative design offers a systematised means by which contextual elements can be traced and accounted for in relation to not only psychological but more broadly, scientific research (Van Bavel et al., 2016).

\section{Conclusion}

Poropat and Corr (2015) point out that not infrequently the same self-report items are featured in analyses notionally at different levels of evolutionary mechanisms, such as at endotype versus phenotype levels of analysis. Such a conflict remains problematic for meaningful discourse regarding the relationship between variables at these different levels, and thus for personality as a feature of human beings. In their words, "adequate integration of personality models will require reliable assessment and analysis of intra- and inter-individual processes and variation, combined with attention to both the expression and perception of personality" (Poropat \& Corr, 2015, p. 66). Generalisability theory offers a first step in improving awareness of intra and inter-individual aspects of the study situation, but it does not address the deeper concern of how evolutionary mechanisms have played a role in the expression of personality traits that can be tracked in a study context. Brunswik's $(1952,1956)$ conceptual framework asks us to account for the impact of several researcher decisions - the choices of variables to represent phenomena, manner of variation within variables, manner of concomitant variation between variables and the effect of variables on each other, and the connection to the environment in terms of whether they are artificially tied together, artificially given to interlock, or artificially untied must be systematically stated. The impact of researcher presences in social relations to participants also has a place for analysis in both generalisability theory and conceptual framework approaches, with the added advantage in a conceptual framework of being matched in some way back to the environment most at question for the sample pool of subjects and presumably, their population.

Brunswik (1955) states psychologists enacted a "double standard" in their inductive inferences - 
we carefully specify our inductive generalisations for participants, but pay no heed to inductive generalisations for environments. Following the outcomes of the 2015 replication crisis for psychology, we are well served today to shift our research practices in the direction of more detailed methodology and rigorous reconciliation of the study context to the environment within which the personality phenomena is given to occur. As big data approaches represent the opportunity to confirm it, we may in time come to understand confirmation of ecological validity as logically preceding tests of reliability, for explanatory outcomes from any given study.

\section{References}

Arterberry, B. J., Martens, M. P., Cadigan, J. M., \& Rohrer, D. (2014). Application of generalizability theory to the Big Five inventory. Personality and Individual Differences, 69, 98-103.

Boag, S. (2011). Explanation in personality psychology: "Verbal magic" and the five-factor model. Philosophical Psychology, 24, 223-243.

Borsboom, D. (2005). Measuring the Mind: Conceptual Issues in Contemporary Psychometrics. Cambridge: Cambridge University Press.

Brunswik, E. (1939). The conceptual focus of some psychological systems. Erkenntnis, 8, 3649.

Brunswik, E. (1952). The Conceptual Framework of Psychology. Chicago: Chicago University Press.

Brunswik, E. (1955). In defense of probabilistic functionalism: A reply. Psychological Review, 62, 236-242.

Brunswik, E. (1956). Perception and the Representative Design of Experiments. Berkeley: University of California Press.

Brunswik, E. (1957). Scope and aspects of the cognitive problem. Contemporary Approaches to Cognition, 5-31.

Tolman, E. C., \& Brunswik, E. (1935). The organism and the causal texture of the environment. Psychological Review, 42, 43-77.

Cramer, A. O. J., van der Sluis, S., Noordhof, A., Wicherts, M., Geschwind, N., Aggen, S. H., Kendler, K. S., \& Borsboom, D. (2012). Dimensions of normal personality as networks in search of equilibrium: You can't like parties if you don't like people. European Journal of Personality, 26, 414-431.

Cronbach, L.J., Rajaratnam, N., \& Gleser, G.C (1963). Theory of generalizability: A liberalization of reliability theory. The British Journal of Statistical Psychology, 16, 137- 
163.

DeYoung, C. G., Hirsh, J. B., Shane, M. S., Papademetris, X., Rajeevan, N., \& Gray, J. R. (2010). Testing predictions from personality neuroscience: Brain structure and the Big Five. Psychological Science, 21, 820-828.

Hammond, K. R. (1966). Probabilistic functionalism. In K.R Hammond (Ed.), The Psychology of Egon Brunswik, (pp. 15-80). New York: Holt, Rinehart \& Winston.

Kosinski, M., Stillwell, D., \& Graepel, T. (2013). Private traits and attributes are predictable from digital records of human behavior. Proceedings of the National Academy of Sciences, 110, 5802-5805.

Lewontin, R. C. (2006). The analysis of variance and the analysis of causes. International Journal of Epidemiology, 35, 520-525.

Machado, A., \& Silva, F. J. (2007). Toward a richer view of the scientific method: The role of conceptual analysis. American Psychologist, 62, 671-681.

Marsh, T., \& Boag, S. (2014). Unifying psychology: Shared ontology and the continuum of practical assumptions. Review of General Psychology, 18, 49-59.

Nowland, T. (2014). Realism, Ontology and Latent Variables. Unpublished Masters thesis.

Petocz, A., \& Mackay, N. (2013). Unifying psychology through situational realism. Review of General Psychology, 17, 216-223.

Petocz, A., \& Newbery, G. (2010). On conceptual analysis as the primary qualitative approach to statistics education research in psychology. Statistics Education Research Journal, 9, 123-145.

Poropat, A. E., \& Corr, P. J. (2015). Thinking bigger: The Cronbachian paradigm \& personality theory integration. Journal of Research in Personality, 56, 59-69.

Rozeboom, W. W. (1977). Metathink: A radical alternative. Canadian Psychological Review, 18, 197-203.

Saucier, G., \& Goldberg, L. R. (2001). Lexical studies of indigenous personality factors: Premises, products and prospects. Journal of Personality, 69, 847-879.

Shavelson, R. J., \& Webb, N. M. (1991). Generalizability Theory: A Primer. London: Sage Publications.

Tolman, E. C., \& Brunswik, E. (1935). The organism and the causal texture of the environment. 
Psychological Review, 42, 43-77.

Van Bavel, J. J., Mende-Siedlecki, P., Brady, W. J., \& Reinero, D. A. (2016). Contextual sensitivity in scientific reproducibility. Proceedings of the National Academy of Sciences, 201521897.

Wood, D., Gardner, M. H., \& Harms, P. D. (2014). How functionalist and process approaches to behavior can explain trait covariation. Psychological Review, 122, 84-111. 